\title{
BUTIN ATTENUATES BRAIN EDEMA IN A RAT MODEL OF INTRACEREBRAL HEMORRHAGE BY ANTI INFLAMMATORY PATHWAY
}

\begin{abstract}
Background: This study evaluates the effect of butin against brain edema in intracerebral hemorrhage (ICH). Methodology: ICH was induced by injecting bacterial collagenase in the brain and all the animals were separated into four groups such as control group, ICH group treated with vehicle, Butin 25 and $50 \mathrm{mg} / \mathrm{kg}$ group receives butin ( 25 and $50 \mathrm{mg} / \mathrm{kg}$, i.p.) $60 \mathrm{~min}$ after the induction of ICH in all animals. One day after neurological score hemorrhagic injury and expressions of protein responsible for apoptosis and inflammatory cytokines were assessed in the brain tissue of ICH rats. Result: Neurological scoring significantly increased and hemorrhagic lesion volume decreased in butin treated group of rats compared to ICH group. However, treatment with butin significantly decreases the ratio of $\mathrm{Bax} / \mathrm{Bcl}-2$ and protein expression of $\mathrm{Cleaved}$ caspase- 3 than ICH group in dose dependent manner. Level of inflammatory mediators such as tumor necrosis factor- $a$ (TNF- $a$ ) and interlukin- 6 (IL-6) in the brain tissues were significantly decreased in the butin treated group than ICH group. In addition butin attenuates the altered signaling pathway of NF-KB in the brain tissues of ICH rats. Conclusion: Our study concludes that butin attenuates the altered behavior and neuronal condition in ICH rats by reducing apoptosis and inflammatory response.
\end{abstract}

Keywords

- Brain edema • Butin • Cytokines • Inflammation • Intracerebral hemorrhage
Peiyu Li,

Cheng Jiwu*

Department Of Neurology, The Affiliated First Hospital

Of Jiamusi University, Jiamusi, Heilongjiang, China

Received 01 December 2017 accepted 28 December 2017

\section{Introduction}

Intracerebral hemorrhage (ICH) occurs due to rupture of blood vessels in the brain that causes coagulation of blood in the brain [1]. This deposition of blood on the brain cells causes edema and damage of neuronal cells. Throughout the world ICH is linked with the highest mortality and approximately $10-15 \%$ of patients of stroke occur due to ICH [2]. Cerebral blood flow was reduced due to increase in the intracranical pressure which leads to poor prognosis [3]. Literature reveals that in $\mathrm{ICH}$ deposition of blood on the brain tissue causes loss of neuron [4]. Inflammatory reactions contribute to neuronal damage due to cytotoxic effects of blood content on the neuronal cells [5]. In addition, pro inflammatory cytokines contribute to the development of $\mathrm{ICH}$ and also induces neuronal apoptosis [6]. Current therapies available for the management of $\mathrm{ICH}$ are medicinal and neurological therapies but it won't be able to control brain adequately. So, new therapy is required for the control of post$\mathrm{ICH}$ brain edema. Thus development of a drug for the management of cerebral hemorrhage is required.

$R$. verniciflua was traditionally used in China for the management of several disorders [7] and butin was isolated from R. verniciflua [8]. Literature reveals that butin has a potential estrogenic effect [9]. In addition butin was reported to possess strong antioxidant, antiplatelet and anti-inflammatory activity [1011]. The neuroprotective effects of butin has yet to be evaluated in the $\mathrm{ICH}$ condition. The present study evaluates the effect of butin on brain edema in intracerebral hemorrhage.

\section{Material and methods}

\section{Animal}

Male albino wistar rats (120-150 g) were procured from Shangai Animal House, China.
Animals were stored under the standard condition as per guidelines. All the animals were kept for a period of 7 days for acclimatization to laboratory conditions with free access to normal standard chow diet and tap water. Protocol of this study is approved by the The Affiliated First Hospital Of Jiamusi University, China (AFHJU/IAEC/2017/03) and the given study followed the guidelines of Association for the Assessment and Accreditation of Laboratory Animal Care International (AAALAC) for experimentation and animal use.

\section{Induction of intracerebral}

\section{hemorrhage}

Bacterial collagenase was injected intrastriatally for the induction of $\mathrm{ICH}$ as per the previously reported method [12]. All the animals were placed on the stereotaxic frame and anesthetized with chloral hydrate (3.6\%). the bregma was exposed by making an incision and hole was done at the injection site by 
drilling i.e. $6 \mathrm{~mm}$ in depth below the skull, 0.2 $\mathrm{mm}$ posterior to bregma, $3.0 \mathrm{~mm}$ left lateral to the midline. type VII bacterial collagenase $(0.5$ $\mathrm{U} / \mu \mathrm{L}$ ) was infused at a rate of $0.5 \mu \mathrm{L} / \mathrm{min}$ using syringe pump in to central striatum. Delivery of collagenase was done for the duration of 5 min to prevent the reflux in animal and for 10 min needle was kept as such after the injection. However, in the control group, saline was injected by the same manner.

All animals were separated into four different groups: control group, $\mathrm{ICH}$ groups treated with vehicle, Butin 25 and $50 \mathrm{mg} / \mathrm{kg}$ group receives butin ( 25 and $50 \mathrm{mg} / \mathrm{kg}$, i.p.) $60 \mathrm{~min}$ after the induction of $\mathrm{ICH}$ in all animals. One day after the operation all animals were sacrificed for the assessment of butins' effect on the ICH.

\section{Estimation of neurological score}

One day after the induction of $\mathrm{ICH}$, a neurological score was estimated as per the previously reported method [13]. Neurological scoring was determined by observing six types of behaviors such as response to whisker stimulation, body proprioception, climbing, forepaw outstretching, symmetry in the movement of all four limbs and spontaneous activity. The score was done from 1-3 scale. Maximum and minimum score was in the range of 18 and 3 respectively. Neurological score was estimated before and after induction of $\mathrm{ICH}$.

\section{Estimation of hemorrhagic injury}

Cresyl violet was used to stain the coronal sections at 20 rostral-caudal levels for the determination of injection volume. Estimation of hemorrhagic volume was done by using Image Pro Plus 6.0 software.

\section{Western blot assay}

RIPA lysis buffer was used for the extraction of total protein sample in the brain tissue. BCA protein assay was performed for the estimation of protein content. Electroblotted and SDSPAGE (10\%) onto nitrocellulose membranes was used to separate $40 \mu \mathrm{g}$ of protein per lane. Primary antibodies were incubated with the membrane after blocking it by using skim milk (5\%). Primary antibodies such as antiGAPDH, anti-NF-kB p65, anti-p-NF-kB p65, anticleaved caspase-3, anti-Bcl-2, anti-Bax, anti-IL-6 and anti-TNF-a were used in the study. Later peroxidase-conjugated secondary antibodies were used for the estimation of binding of primary antibodies. chemiluminescence was applied for the estimation of protein by analyzing the band pattern with Quantity One 4.1.0 software.

\section{RT-PCR}

Trizol Reagent was utilized for the extraction of total RNA from the brain tissue and Super RT One Step RT-PCR Kit was used to perform RT-PCR analysis as per the direction given by the manufacturer. Specific primer for TNF-a and $\beta$-actin was sued in the study. Relative quantification of gene expression was performed using the $2^{-\Delta \Delta C t}$ method, with $\beta$-actin mRNA as an internal control.
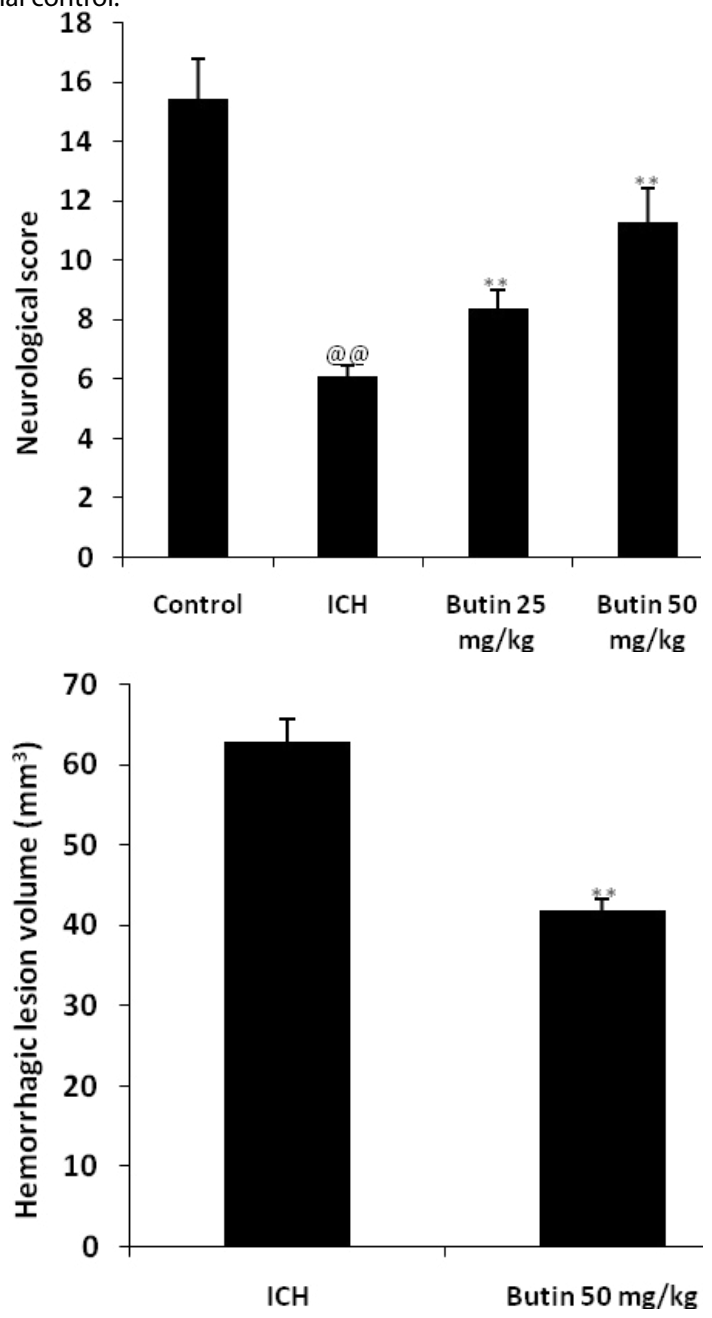

Fig. 1. Effect of butin on the neurological score and hemorrhagic injury was estimated in $\mathrm{ICH}$ rats Values Mean $\pm S D(n=6)$, ${ }^{\oplus} p<0.01$ Vs Control group; ${ }^{* *} p<0.01$ Vs ICH group

\section{Statistical analysis}

All data are shown as mean \pm standard deviation ( $S D, n=10)$, and were analysed by one-way ANOVA, followed by Dunnett's post hoc test (Gradpad prism 6.1., CA, USA). $P<0.05$ was considered statistically significant.

\section{Result}

\section{Effect of butin on the neurological score and hemorrhagic injury}

Effect of butin on the neurological score and hemorrhagic injury in $\mathrm{ICH}$ rats was shown in Fig. 1. There was significant decrease in the neurological scoring in the $\mathrm{ICH}$ group as compared to the control group. However, neurological scoring was significantly 

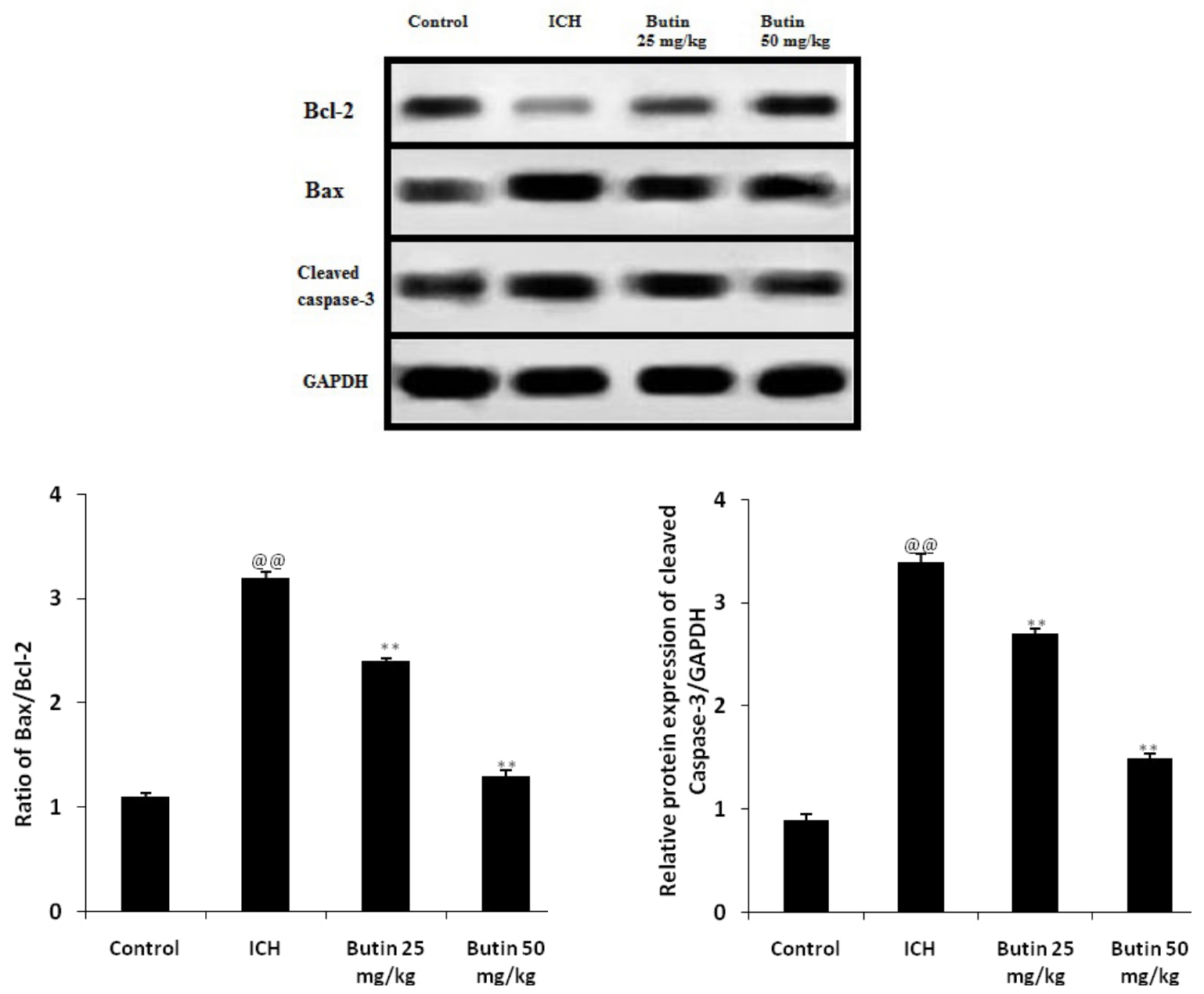

Fig. 2. Effect of butin on the expressions of Bax, Bcl-2 and cleaved caspase- 3 in brain tissues of ICH rats Values Mean \pm SD $(n=6)$, ${ }^{@} p<0.01$ Vs Control group; ${ }^{* *} p<0.01$ Vs ICH group

increased in the butin treated group of rats compared to $\mathrm{ICH}$ group in a dose dependent manner. In addition, hemorrhagic lesion volume was significantly reduced in the butin treated group than ICH group.

\section{Effect of butin on the apoptosis of neuron}

Effect of butin on the expressions of Bax, Bcl2 and cleaved caspase- 3 in brain tissues of $\mathrm{ICH}$ rats was assessed for the estimation of neuronal apoptosis as shown in fig. 2. It was observed that the ratio of $\mathrm{Bax} / \mathrm{BCl}-2$ and protein expression of Cleaved caspase-3 significantly enhanced in $\mathrm{ICH}$ treated group than control group. However, treatment with butin significantly decreases the ratio of $\mathrm{Bax} / \mathrm{Bcl}-2$ and protein expression of Cleaved caspase-3 than ICH group in dose dependent manner.

\section{Effect of butin on the NF- $\mathrm{kB}$ signaling pathway}

Effect of butin on the NF- $\mathrm{KB}$ signaling pathway was estimated by determining the expressions of $\mathrm{p}-\mathrm{NF}-\mathrm{KB}$ p 65 and NF- $\mathrm{kB}$ p56 in brain tissues of $\mathrm{ICH}$ rats as shown in Fig. 3. It was observed that the expressions of $\mathrm{p}-\mathrm{NF}-\mathrm{kB}$ p65significantly enhances as the phosphorylation of $\mathrm{p} 65$ protein is also enhanced in $\mathrm{ICH}$ group compared to control group. The altered expression of $\mathrm{p}-\mathrm{NF}$ KB p65 was significantly attenuated by butin in $\mathrm{ICH}$ rats.

\section{Effect of butin on inflammatory}

\section{mediators}

Effect of butin on the level inflammatory mediators in brain tissues of $\mathrm{ICH}$ rats was shown in Fig. 4. There was significant increase in the expressions of inflammatory mediators such as TNF- $a$ and IL- 6 in ICH group compared to control group of rats. Treatment with butin significantly decreased the expressions of TNF- $a$ and IL- 6 in the brain tissues of ICH rats.

\section{Discussion}

Literature suggests $\mathrm{ICH}$ causes secondary brain injury leading to brain edema [14]. Development of brain edema after $\mathrm{ICH}$ 

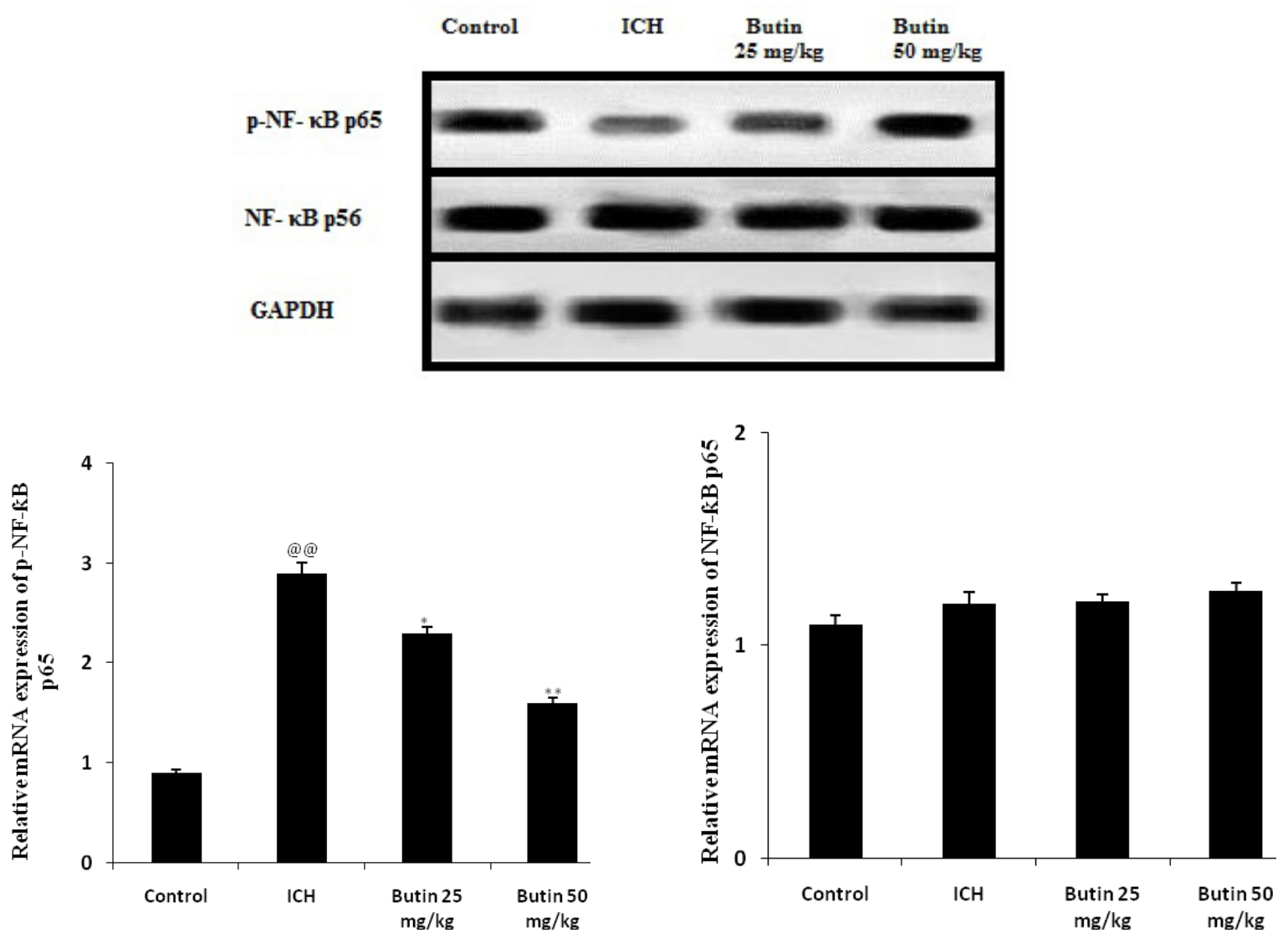

Fig. 3. Effect of butin on the expressions of $p-N F-K B$ p 65 and NF- $K B$ p56 in brain tissues of ICH rats Values Mean $\pm S D(n=6)$, ${ }^{\oplus} p<0.01$ Vs Control group; ${ }^{*} p<0.05,{ }^{* *} p<0.01$ Vs ICH group

causes brain herniation due to increase in the intracranial pressure [15]. Several reports reveal that neurological function is decreased in patients suffering from brain edema [16]. Butin has an estrogenic action and the drugs mimic the action of estrogen's reported neuroprotective effect [17]. In addition to it cyto-protective effect, butin was reported to have anti-inflammatory and antioxidant properties [18]. Our study evaluated the protective effect of butin in $\mathrm{ICH}$ in rats. $\mathrm{ICH}$ was induced by injecting bacterial collagenase in rat brain and several parameters such as neurological score, hemorrhagic volume, expressions of inflammatory mediators and pathway of apoptosis was assessed in the rats' brains.

Neurological scoring decreased in patients suffering of intracranial injury and increased cerebral edema [19]. Previously reported investigations reveals that drugs used for the treatment of $\mathrm{ICH}$ improves brain edema and neurological scoring; and the result of our study also suggests that treatment with butin significantly attenuates the altered level of cerebral edema and neurological score in $\mathrm{ICH}$ rats [20]. $\mathrm{ICH}$ leads to apoptosis of neuronal cells by activating the caspase enzyme cascade due to alteration in the regulation of antiapoptotic protein $\mathrm{BCl}-2$ and pro-apoptotic Bax [21]. Apoptosis of cells is controlled by the caspase 3 enzyme, which is up regulated in the $\mathrm{ICH}$ condition [22]. Data of our study revealed that the ratio of $\mathrm{Bax} / \mathrm{Bcl}-2$ was significantly reduced and the expression of caspase 3 proteins was also decreased in the brain tissues of butin treated rats than $\mathrm{ICH}$ rats.

In addition, the development of secondary brain injury after $\mathrm{ICH}$ occurs due to up regulation of the inflammatory process. The level of pro-inflammatory mediators increased in the $\mathrm{ICH}$ condition and treatment with butin was found to significantly decrease the level of pro-inflammatory cytokines than ICH group. In addition for the inflammatory response NF$\mathrm{KB}$ signaling pathway plays an important role and level of NF-KB elevated in ICH condition. However, butin attenuated the altered level of $\mathrm{NF}-\mathrm{KB}$ in the brain tissues of ICH group.

\section{Conclusion}

In conclusioin, our study found that butin attenuates the altered behavior and neuronal condition in $\mathrm{ICH}$ rats by reducing apoptosis and the inflammatory response. Thus butin shows a promising effect against neurological impairments in the $\mathrm{ICH}$ condition.

\section{Acknowledgement}

Authors of the present study thankful to The Affiliated First Hospital Of Jiamusi University, 

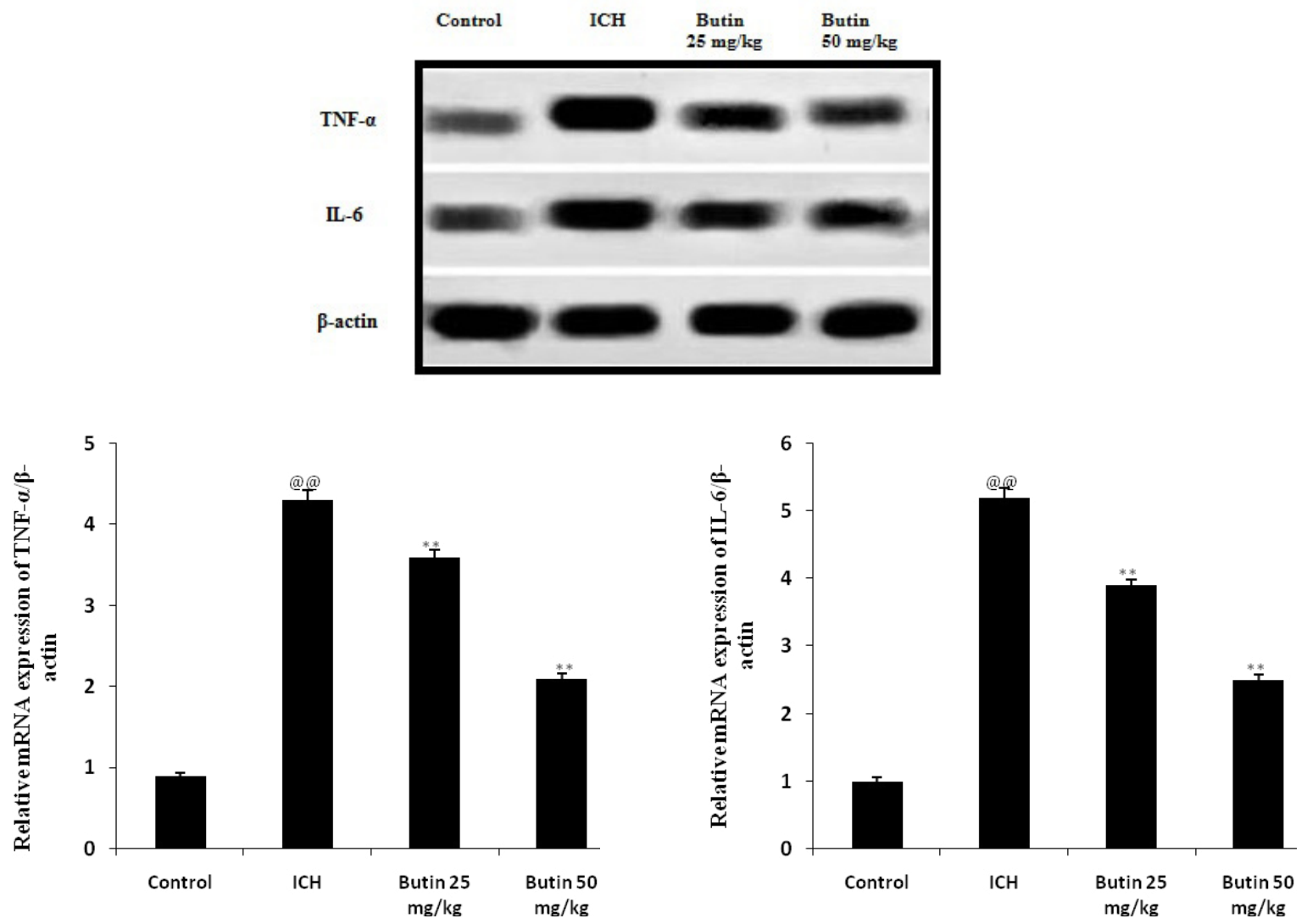

Fig. 4. Effect of butin on the level inflammatory mediators in brain tissues of $\mathrm{ICH}$ rats Values Mean $\pm S D(n=6)$, $₫ p<0.01$ Vs Control group; ${ }^{* *} p<0.01$ Vs ICH group

China for providing fund and necessary facility to complete this project.

\section{Conflict of interest}

There was no conflict of interest

\section{Author's contribution}

Cheng Jiwu designed the protocol, supervise the work and write the manuscript. Peiyu Li perform the experimental work along with statistical analysis and histopathology study.

\section{References}

[1] van Asch CJ, Luitse MJ, Rinkel GJ, van der Tweel I, Algra A, Klijn CJ. Incidence, case fatality, and functional outcome of intracerebral haemorrhage over time, according to age, sex, and ethnic origin: a systematic review and meta-analysis. Lancet Neurol 2010; 9: 167- 76.

[2] Krishnamurthi RV, Feigin VL, Forouzanfar MH, Mensah GA, Connor M, Bennett DA, et al. Global and regional burden of first-ever ischaemic and haemorrhagic stroke during 1990-2010: findings from the Global Burden of Disease Study 2010. Lancet Glob Health 2013; 1: e259- 81.

[3] Arima H, Wang JG, Huang Y, Heeley E, Skulina C, Parsons MW, et al. Significance of perihematomal edema in acute intracerebral hemorrhage: the INTERACT trial. Neurology 2009; 73: 1963-8.
[4] Thiex R, Tsirka SE. Brain edema after intracerebral hemorrhage: mechanisms, treatment options, management strategies, and operative indications. Neurosurg Focus 2007; 22: E6.

[5] Inaji, M., H. Tomita, O. Tone, et al. 2003. Chronological changes of perihematomal edema of human intracerebral hematoma. Acta Neurochirurgica. Supplement 86: 445-448.

[6] Gebel Jr., J.M., E.C. Jauch, T.G. Brott, et al. 2002. Relative edema volume is a predictor of outcome in patients with hyperacute spontaneous intracerebral hemorrhage. Stroke 33(11): 26362641.

[7] Fu SY, Wei SN, Hua ZH, the research progress of biological form $R$. verniciflua strokes, Chinese wild plant. Resources 2005; 24: 12-16. 
[8] Park MH, Kim IS, Kim SA, Na CS, Hong CY, Dong MS, Yoo HH, Inhibitory effect of Rhus verniciflua Stokes extract on human aromatase activity; butin is its major bioactive component. Bioorg Med Chem Lett. 2014 Apr 1;24(7):1730-3.

[9] Bhargava SK. Estrogenic and postcoital anticonceptive activity in rats of butin isolated from Butea monosperma seed. J Ethnopharmacol. 1986 Oct;18(1):95-101.

[10] Lee, M. H., Lin, Y. P., and Hsu, F. L. (2006). Bioactive constituents of Spatholobussub erectus in regulating tyrosinase-related proteins and mRNA in HEMn cells. Phytochemistry 67, 1262-1270.

[11] Kang, K. A., Lee, J. H., and Chae, S. (2009). "Butin decreases oxidative stress-induced 8-hydroxy-20-deoxyguanosine levels via activation of oxoguanine glycosylase," Chem. Biol. Interact 181, 338-342.

[12] Lee ST, Chu K, Jung KH, Kim SJ, Kim DH, Kang KM, Hong NH, Kim JH, Ban JJ, Park HK, Kim SU, Park CG, Lee SK, Kim M, Roh JK. Anti-inflammatory mechanism of intravascular neural stem cell transplantation in haemorrhagic stroke. Brain 2008; 131: 616-629.

[13] Chen J, Li Y, Wang L, Zhang Z, Lu D, Lu M, Chopp M. Therapeutic benefit of intravenous administration of bone marrow stromal cells after cerebral ischemia in rats. Stroke 2001; 32: 1005-1011.

[14] Zamzow DR, Elias V, Legette LL, Choi J, Stevens JF, Magnusson KR. Xanthohumol improved cognitive flexibility in young mice. Behav Brain Res 2014; 275: 1-10.

[15] Panickar KS, Jang S. Dietary and plant polyphenols exert neuroprotective effects and improve cognitive function in cerebral ischemia. Recent Pat Food Nutr Agric 2013; 5: 128-143.
[16] Yao J, Zhang B, Ge C, Peng S, Fang J. Xan-thohumol, a Polyphenol Chalcone Present in Hops, Activating Nrf2 Enzymes To Confer Protection against Oxidative Damage in PC12 Cells. J Agr Food Chem 2015; 63: 1521-1531.

[17] Cho N, Choi JH, Yang H, Jeong EJ, Lee KY, Kim YC, Sung SH. Neuroprotective and anti-inflammatory effects of flavonoids isolated from Rhus verniciflua in neuronal HT22 and microglial BV2 cell lines. Food Chem Toxicol. 2012 Jun;50(6):1940-5.

[18] RuiZhangaKyoung AhKangaMei JingPiaoaWeon YoungChangaYoung HeeMaengaSungwookChaebln KyungLeecBum JoonKimcJin WonHyun, Butin reduces oxidative stress-induced mitochondrial dysfunction via scavenging of reactive oxygen species. Food and Chemical Toxicology, 48, Issue 3, March 2010, Pages 922-927.

[19] Smaili SS, Hsu YT, Sanders KM, Russell JT, Youle RJ. Bax translocation to mitochondria subsequent to a rapid loss of mitochondrial membrane potential. Cell Death Differ 2001; 8: 909-920.

[20] Tawa P, Hell K, Giroux A, Grimm E, Han Y, Nicholson DW, Xanthoudakis S. Catalytic activity of caspase- 3 is required for its degradation: stabilization of the active complex by synthetic inhibitors. Cell Death Differ 2004; 11: 439-447.

[21] Simard JM, Geng Z, Woo SK, Ivanova S, Tosun C, Melnichenko L, Gerzanich V. Glibenclamide reduces inflammation, vasogenic edema, and caspase-3 activation after subarachnoid hemorrhage. J Cereb Blood Flow Metab 2009; 29: 317-330.

[22] Wasserman JK, Schlichter LC. Neuron death and inflammation in a rat model of intracerebral hemorrhage: effects of delayed minocycline treatment. Brain Res 2007; 1136: 208-218. 